\title{
Motor imagery for pain and motor function after spinal cord injury: a systematic review
}

\author{
Emmanuelle Opsommer ${ }^{1} \cdot$ Odile Chevalley $^{1} \cdot$ Natalya Korogod $^{1}$
}

Received: 20 March 2019 / Revised: 17 November 2019 / Accepted: 21 November 2019 / Published online: 13 December 2019

(c) The Author(s), under exclusive licence to International Spinal Cord Society 2019

\begin{abstract}
Study design Systematic review.

Objectives To evaluate the therapeutic benefits of motor imagery (MI) for the people with spinal cord injury (SCI). Setting International.

Methods We searched electronic bibliographic databases, trial registers, and relevant reference lists. The review included experimental and quasi-experimental study designs as well as observational studies. For the critical appraisal of the 18 studies retrieved (three RCT, seven quasi-RCT, eight observational), we used instruments from the Joanna Briggs Institute. The primary outcome measure was pain. Secondary outcome measures included motor function and neurophysiological parameters. Adverse effects were extracted if reported in the included studies. Because of data heterogeneity, only a qualitative synthesis is offered.

Results The included studies involved 282 patients. In most, results were an improvement in motor function and decreased pain; however, some reported no effect or an increase in pain. Although protocols of MI intervention were heterogeneous, sessions of 8-20 min were used for pain treatments, and of 30-60 min were used for motor function improvement. Neurophysiological measurements showed changes in brain region activation and excitability imposed by SCI, which were partially recovered by MI interventions. No serious adverse effects were reported.

Conclusions High heterogeneity in the SCI population, MI interventions, and outcomes measured makes it difficult to judge the therapeutic effects and best MI intervention protocol, especially for people with SCI with neuropathic pain. Further clinical trials evaluating MI intervention as adjunct therapy for pain in SCI patients are warranted.
\end{abstract}

\section{Introduction}

Pain is a common complication after spinal cord injury (SCI), which can be related to effects of the spinal injury, a SCI-imposed lifestyle, or to pre-existing conditions. The most common pain types are nociceptive and neuropathic pain $(\mathrm{NeP})$ [1-3]. Management of chronic pain after SCI is very challenging [4] and recent reviews conclude that there

Supplementary information The online version of this article (https:// doi.org/10.1038/s41393-019-0390-1) contains supplementary material, which is available to authorized users.

Emmanuelle Opsommer

emmanuelle.opsommer@hesav.ch

1 School of Health Sciences (HESAV) - University of Applied Sciences and Arts Western Switzerland (HES-SO), Avenue de Beaumont 21, 1011 Lausanne, Switzerland is still a lack of evidence for the impact of both pharmacological and non-pharmacological treatments [5].

Recent studies have shown that motor cortex stimulation can be used as one of the non-pharmacological approaches to treat pain $[6,7]$. It has been proposed that cortical structures involved in movement control might be reorganized $[8,9]$ as a consequence of the spinal cord lesion causing a mismatch between motor output and sensory feedback [10]. These changes in turn could lead to the pain experience [11]. Correcting this discord between mental body representation, sensory-motor integration, and nociception may help in chronic pain treatment [12].

Motor imagery (MI) is one of the techniques which could be used for this purpose. It is defined as mental representation of movement without any actual body movement or peripheral muscle activation [13-16]. The brain areas (including motor cortex) active during MI and movement itself are largely overlapping [17]. This could explain the fact that mental movement repetition, especially when 
combined with physical practice, improves motor performance in healthy people [18], athletes [19] as well as in individuals with neurological disorders, including SCI [13, 14, 20-22]. However, the effect of MI interventions on pain remains unclear: some studies showed a reduction $[10,23]$, some an increase [24] and some no effect on pain [25]. There could be many reasons underlying these discrepancies, such as patient's perception of pain, social stressors, patient's expectation from treatment, or MI methodology itself $[12,26]$. Indeed, there are different ways to perform MI. It can be carried out from two perspectives: external (third-person) or internal (first-person). The thirdperson perspective is an imagery where a "person views him- or herself from the perspective of an external observer" (i.e., seeing him/herself performing the imagined movement). It is considered to be mainly visual in nature. The first-person perspective requires the person to imagine "being inside his/her body and experiencing those sensations" as if he/she was performing the movement. Therefore, internal (first-person) imagery may include both visual and kinesthetic components [15 p. 945]. Imagery capacity may differ from person to person and should be tested before performing an MI intervention, especially in people with neurological deficits [8]. For example, it was shown that individuals with SCI have difficulties performing MI from the first-person perspective [27]. Several tools exist to assess imagery ability and one of them, the Kinesthetic and Visual Imagery Questionnaire (KVIQ), is especially adapted for individuals with disabilities [28].

To answer questions on the therapeutic benefits of MI interventions on pain in SCI we performed a preliminary search in several databases. The review by Aikat and Dua [29] discussed the therapeutic potential of MI interventions in SCI, but without a critical appraisal of the evidence and not specifically addressing the aspect of pain. The primary purpose of our systematic review was to provide a scrupulous summary of all available primary research on the therapeutic effects of MI interventions on pain in individuals with SCI. A secondary aim was to investigate effects on motor function recovery. Where possible, we extracted information about neurophysiological changes associated with brain activity during MI and discussed the details of MI protocols (i.e., frequency, intensity, duration) for both pain and motor recovery treatments in patients with SCI.

\section{Methods}

We published a protocol prior to undertaking this review [12] which was registered with PROSPERO (\# CRD42017075144). This systematic review was conducted in accordance with the Joanna Briggs Institute (JBI) methodology for systematic review of effectiveness evidence
[30]. The search strategy, examples of search algorithms with keywords and index terms, as well as information about data extraction procedures, are provided in the Supplementary File 1.

\section{Inclusion and exclusion criteria}

This review considered both experimental and quasiexperimental designs as well as observational studies published as full text in English, French, or German (Fig. 1). Studies in other languages were excluded following title appraisal. Studies must have involved primarily adults (18 years and older) with a diagnosis of SCI [12] and have evaluated a MI intervention provided as an independent intervention, added to other therapy, or embedded in therapy. Primary outcomes were those related to pain [31], such as pain intensity (assessed with Visual Analog Scale (VAS), Numerical Rating Scale (NRS), Brief Pain Questionnaire etc.) and pain duration. Secondary outcomes were motor function and activity/disability related outcomes. Additional outcomes were neurophysiological measures of brain activity (i.e., functional magnetic resonance imaging (fMRI), positron emission tomography, electroencephalography (EEG) and magnetoencephalography (MEG)) and motor output (e.g., motor evoked potentials and motor thresholds).

\section{Study selection}

A three-step search strategy was used [12]: (1) initial search in PubMed and CINAHL with text word analysis of the title and abstract to identify the keywords and descriptors; (2) secondary search in all databases (JBI Database of Systematic Reviews and Implementation Reports, Cochrane, PubMed, Embase, CINAHL, PsychINFO, PEDro, OTseeker, Campbell, DARE, TRIP, NICE, and BestBets and Bandolier) with identified keywords and index terms; (3) reference lists of all identified reports and articles were searched for additional studies. Individual search strategies were developed for each database to take into account any differences in thesaurus terminology and indexing (example for PubMed in Supplementary file 1). Examples of keywords used: (Spinal Cord Injury OR Spinal Cord Injuries OR Spinal Cord Ischemia OR Paraplegia OR Quadriplegia) AND (Imagery OR Imagination OR Mental Practice OR Cognitive rehearsal OR Guided Imagery OR Motor Imagery). All citations identified were loaded into EndNote ${ }^{\mathrm{TM}}$ and duplicates removed. Titles and then abstracts were screened by two reviewers independently against the inclusion criteria for the review (Fig. 1). The full text of potentially eligible studies was retrieved and assessed against the inclusion criteria by two reviewers independently. Any disagreements that arose between them were resolved through discussion, or with a third reviewer casting a deciding vote. 
Fig. 1 PRISMA flow diagram describing screening and review process. *Until 31.01.2019.

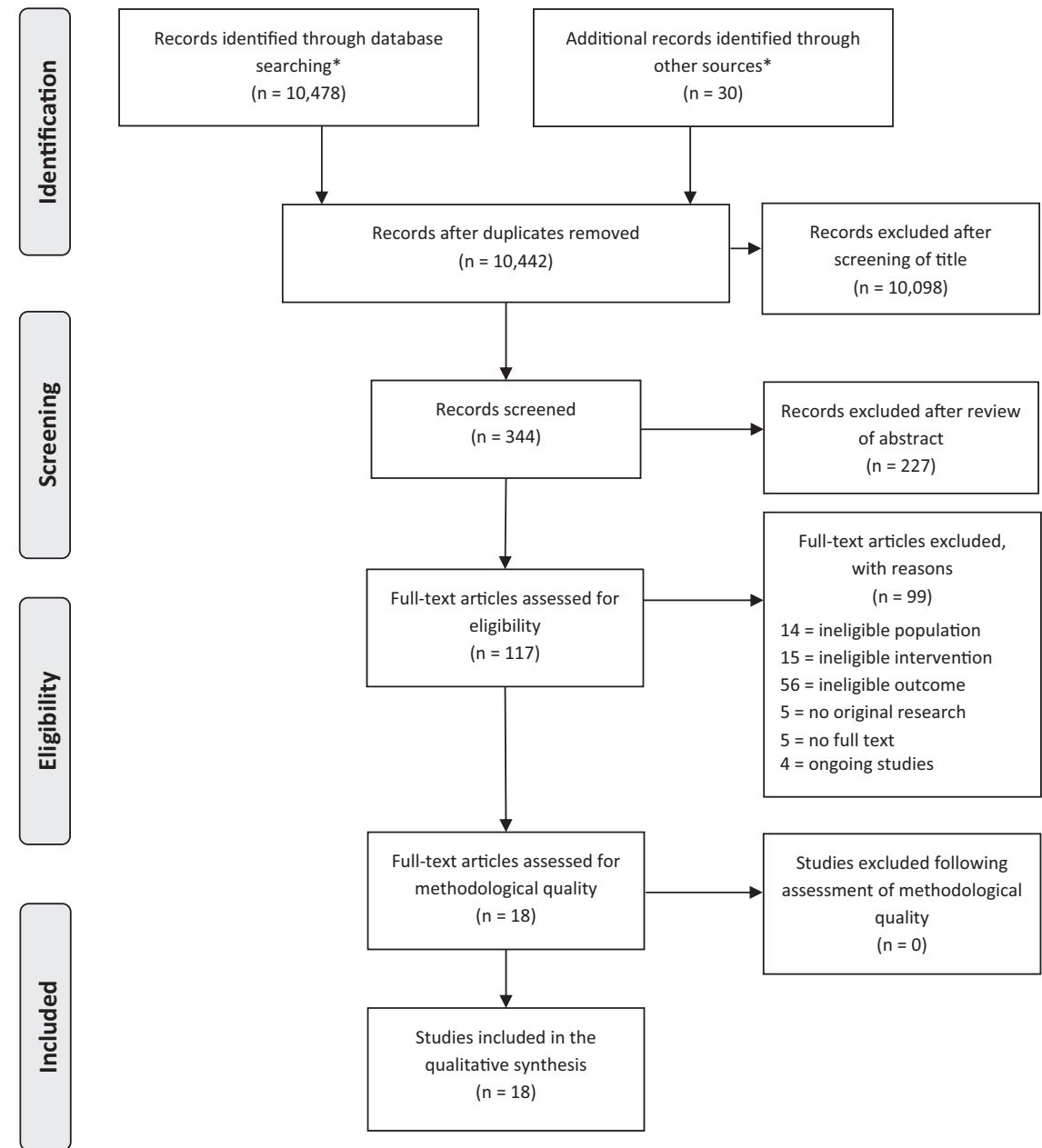

analysis. The effect size was the mean difference between measures of pain intensity. For this, we used Review Manager (RevMan. Version 5.3. Copenhagen: The Nordic Cochrane Centre, The Cochrane Collaboration, 2014).

\section{Results}

Figure 1 is the PRISMA flow diagram. Electronic bibliographic databases and additional data source searching returned 10,442 non-duplicate titles. Following title and abstract screening, we screened 117 in full text. Eighteen studies were eligible for the review and critically appraised $[10,11,20,21,23-25,32-42]$.

\section{Methodological quality}

All studies met the minimum established criteria of moderate to high quality (Supplementary Tables 1-4). Overall, the most frequent risk was selection bias as the majority of the studies did not use randomization for treatment allocation, or did not use a control group. 


\section{Characteristics of included studies}

Table 1 describes the population, intervention and comparator as well as the outcomes of the studies included.

\section{Types of studies}

Three RCT studies compared two groups of people with SCI [23, 38, 42]. Seven further experimental studies compared either persons with SCI with and/or without NeP to healthy participants, or persons with SCI with $\mathrm{NeP}$ to those without $\mathrm{NeP}$ [11, 20, 24, 34, 35, 40-42]. Five studies were case series $[10,25,36,37,39]$ and three were case reports $[21,32,33]$.

\section{Participants}

The 18 studies involved 282 participants with SCI (mean age: $44.3 \pm 11.4$ years, range $21-72$ ). The majority of participants with SCI were male (78\%). All studies but one [37] (patients in subacute SCI phase with average time after injury of 50 days) included patients with SCI at a chronic stage, with an average time since injury of $7.3 \pm 6.1$ years (range 0.3-40). The neurological level of injury (NLI) was between C3 and L3 with complete or incomplete lesions. Among individuals with SCI, NeP was present in 166 people and nociceptive pain in 17; a patient may have had both nociceptive and NeP at the start of the study. To classify pain, some studies [10, 11, 23, 24, 33, 34, 40-42] referred to the taxonomy of SCI pain by Siddall et al. [43]; one study [42] referred to the Bryce-Ragnarsson Pain Classification Scheme [44]. Pain was reported as a characteristic of the population, as an inclusion criterion [10, 11, 23-25, 27, 33, 34, 39-42], an exclusion criterion [38], a limitation for MI [32], an outcome measure [10, 11, 23-25, 27, 33, 34, 37, 39-42], an adverse event $[11,24]$ or not reported at all $[20,21,35,36]$. There was high heterogeneity in participants with SCI, who were different in years since lesion, NLI and completeness of injury and type of pain.

\section{Interventions}

The imagery capacity of the participants with SCI was tested in three out of 18 studies only, by using KVIQ [25, 32, 35]. Different MI protocols were applied in the studies included. MI interventions were applied with audiotape support $[11,20,24,38]$, under supervision $[21,32,35]$, or in combination with brain computer interface (BCI) [36, 37, 40, 41]. Other studies used virtual walking training, which required the participants to imagine performing the movements they were shown, as a standalone intervention $[10,25,33,42]$ or in combination with transcranial direct current stimulation (tDCS) [23, 34]. One study [39] applied mirror visual feedback in which patients while looking at the reflected image of their non-paralyzed/ unaffected limb in the mirror (occupying the space of their paralyzed/affected or phantom limb) had to perform or imagine the movements of both non-paralyzed/unaffected limb and non-paralyzed/affected or phantom limb.

The duration of sessions varied from 8 min per day to 60 min per day, and total treatment length varied from 1 to 84 days. Follow-up assessments were performed at 1 [21], 2 [35], 3 [23, 32, 33, 38], and twelve months [36].

\section{Outcome measures}

The most common measures were pain intensity, measured with VAS and NRS [10, 11, 23-25, 33, 34, 39-42]. Further pain measures were the location of pain $[10,11,23,24,34,40]$, pain quality (description of painsuperficial or deep [39], McGill Pain Questionnaire $[10,37]$, a scale inspired by the McGill Pain Questionnaire [27], Neuropathic Pain Scale (NPS) [42], Neuropathic Pain Symptom Inventory [23]), and the temporal aspects of pain such as the duration of pain relief [10]. The Brief Pain Inventory (BPI) [23, 40], the Basic Pain Data Set [25], the Hospital Anxiety and Depression Scale [25], and the Patient's Global Impression of Change (PGIC) [23] were also reported.

Motor component and activity/disability outcomes were assessed with various tests: gait velocity [38], Performance Oriented Mobility Assessment [38], SCI independence measure [38], muscle strength [20,35], rate of movement [20], kinematics of upper limb [21, 32, 35], Box and Block test [32, 35], Minnesota Manual Dexterity Test [32, 35], muscle strength [21], and Functional Independent Measure [21].

Neurophysiological measurements of brain activity during MI were done in six quasi-experimental $[11,20,34,35,40,41]$ and three observational studies $[32,36,37]$. Two studies used fMRI [11, 20], four EEG [36, 37, 40, 41] and one MEG [35].

\section{Review findings}

Table 2 presents a summary of the pain intensity outcomes, the motor function and activity/disability related outcomes.

\section{Pain outcomes}

The effects of MI interventions on pain severity are conflicting. Studies using visual illusion combined with MI (Fig 2.1), showed either an improvement after the intervention [10, 23, 33, 34, 39, 42] or no effect [25]. Two studies by Gustin et al. using MI supported by audiotape 


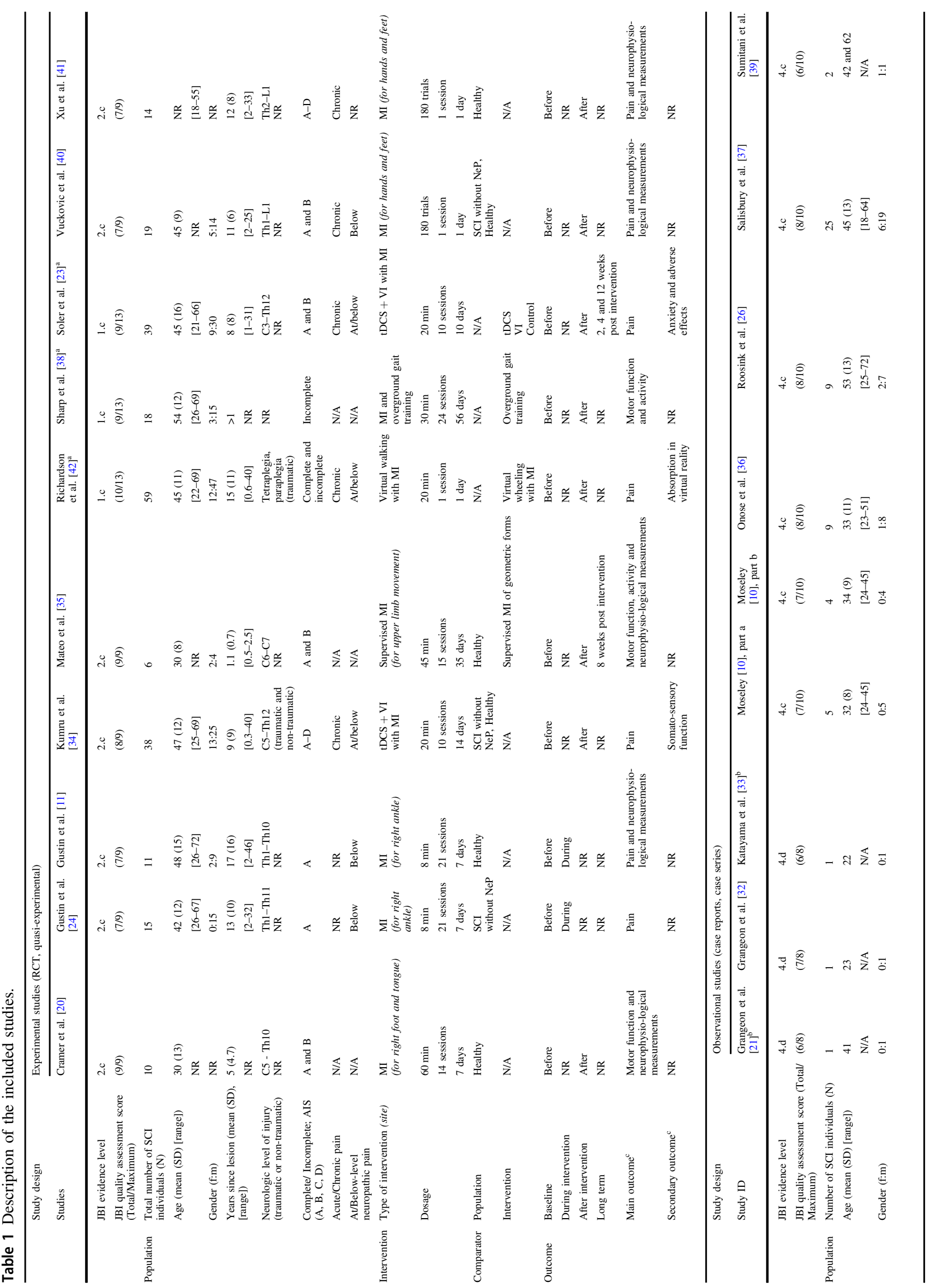




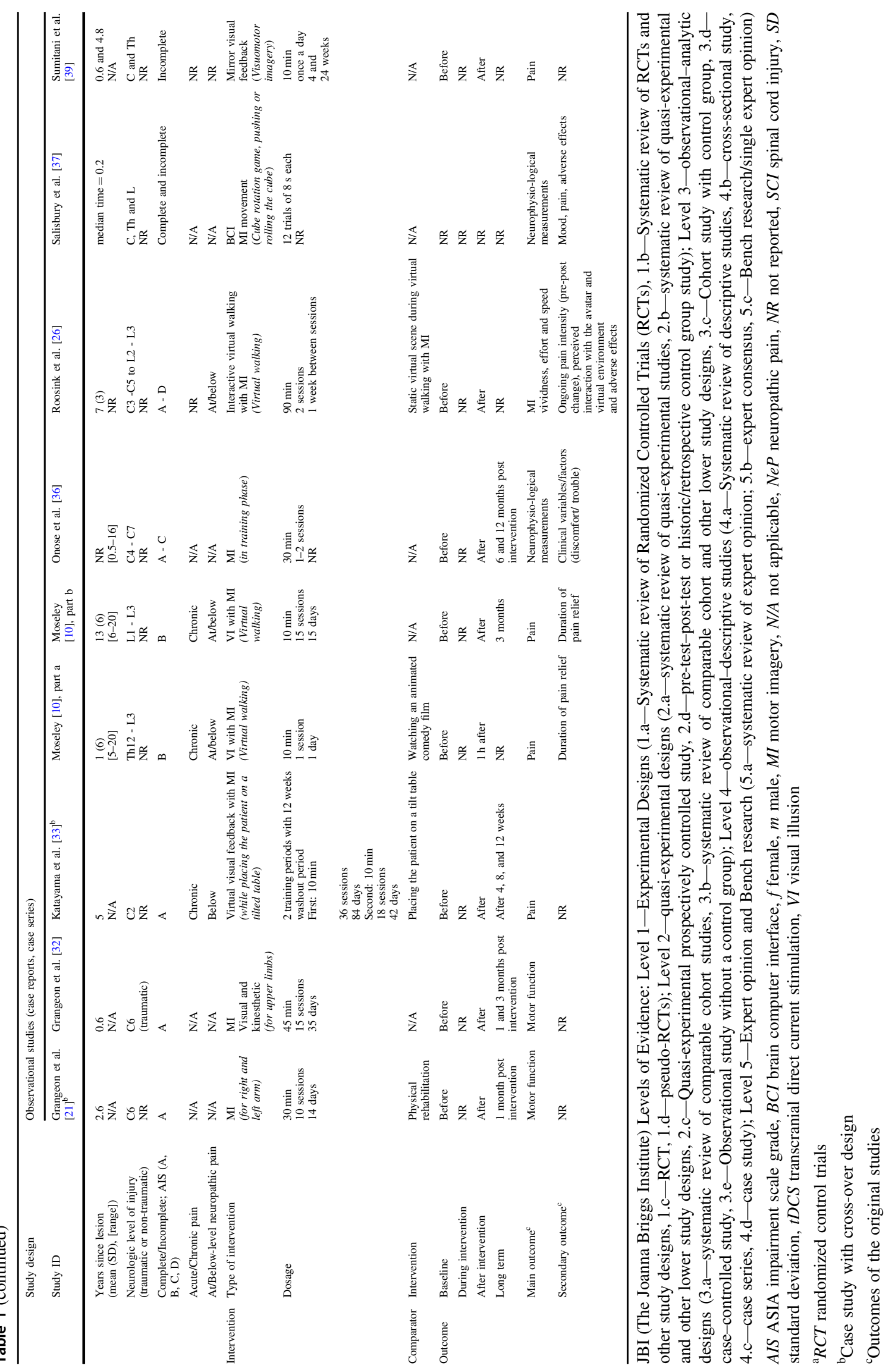




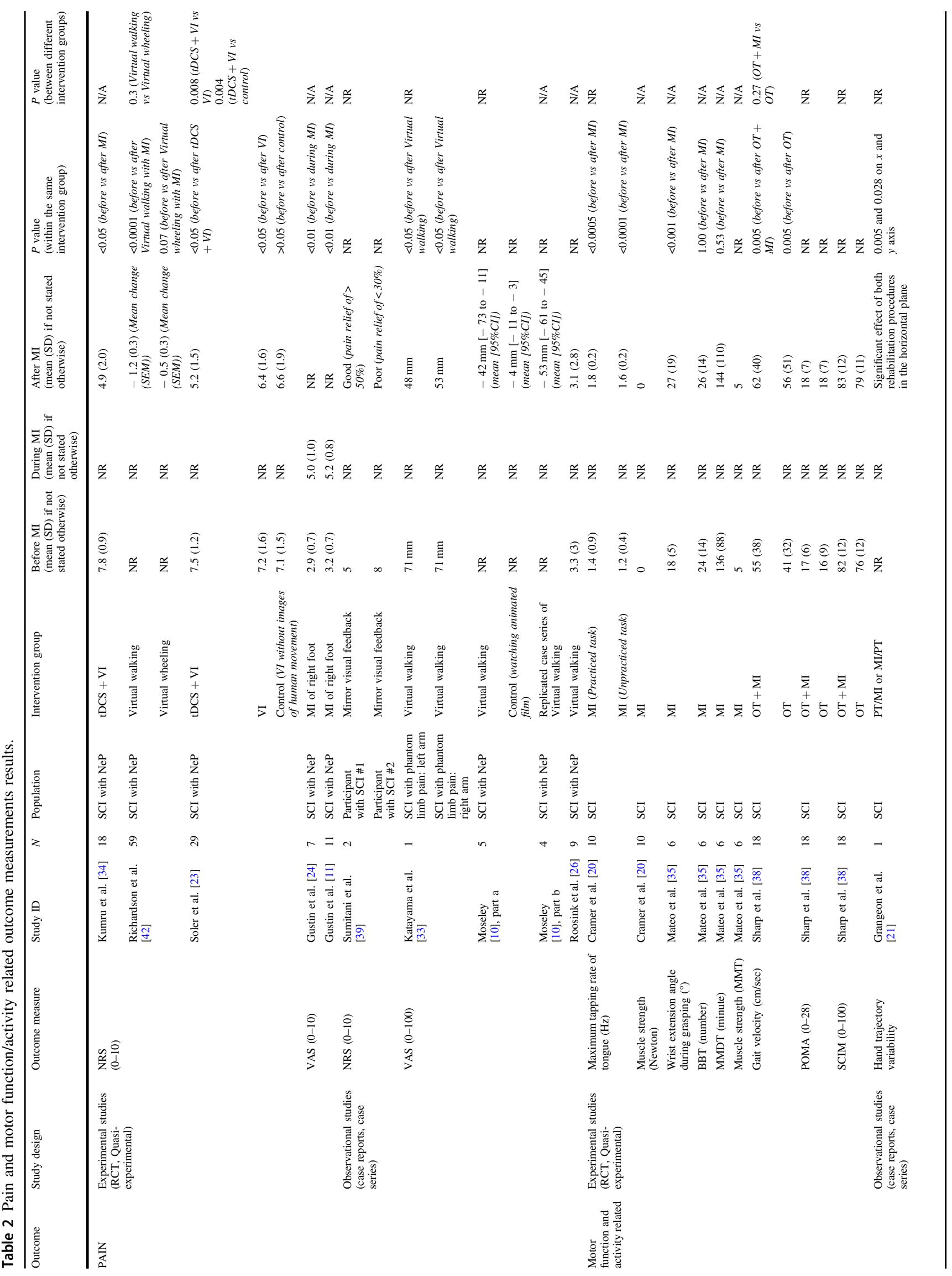




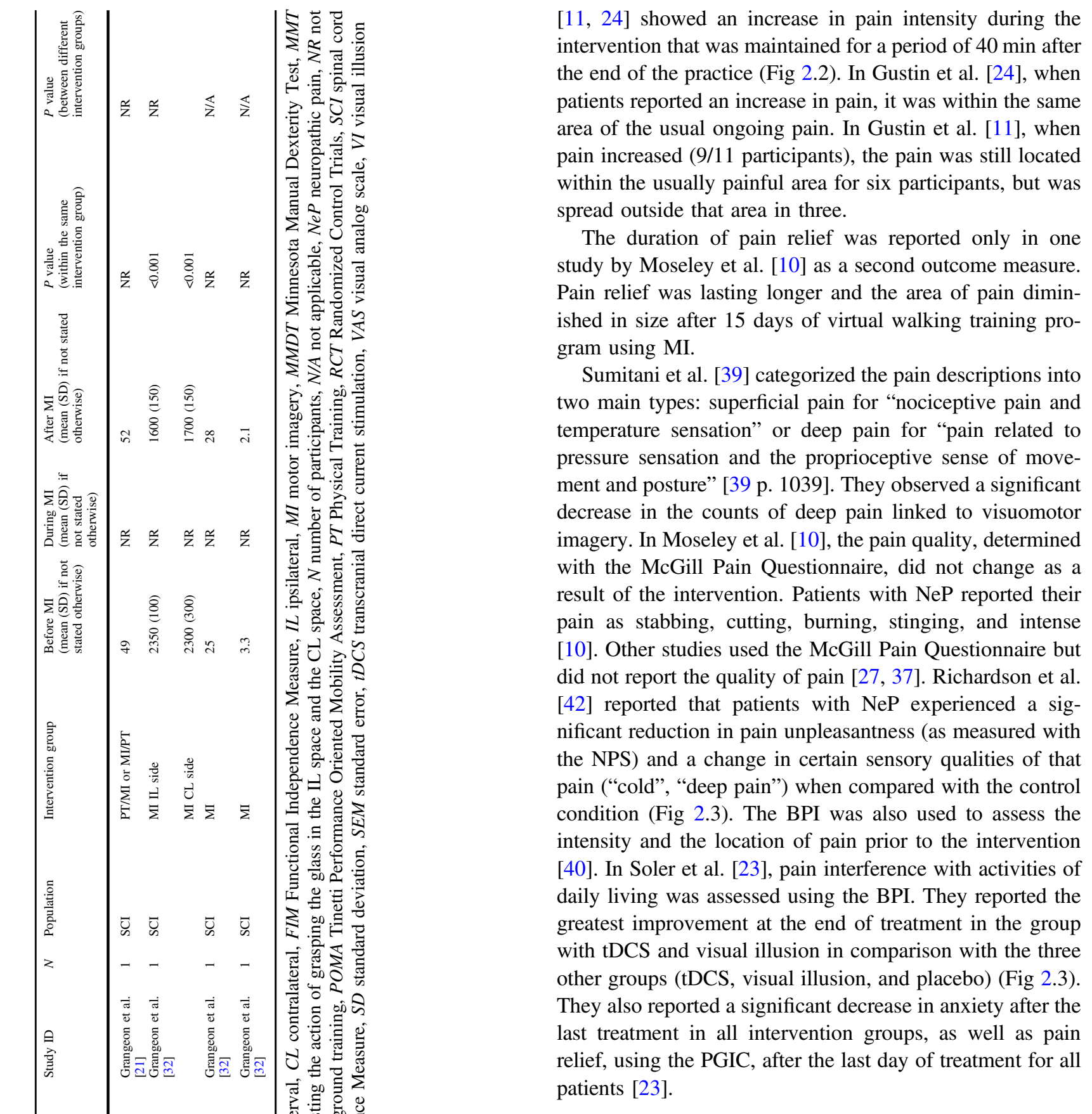

Motor function and activity/disability outcomes

Motor function and activity/disability outcomes were assessed in five studies [20, 21, 32, 35, 38]. Conventional therapy was used in addition to MI in all five. One study [38] assessed lower limb function and four [20, 21, 32, 35] upper limb function. Because of high heterogeneity of the studies, the data for motor function and activity/disability outcomes are presented in narrative form in Table 2.

Cramer et al. [20] showed that 1 week of MI training produced greater gains on maximum physical tapping rate of tongue and right foot, for a practiced than for an 
2.1 Comparing effect of MI on pain intensity at baseline and post-treatment

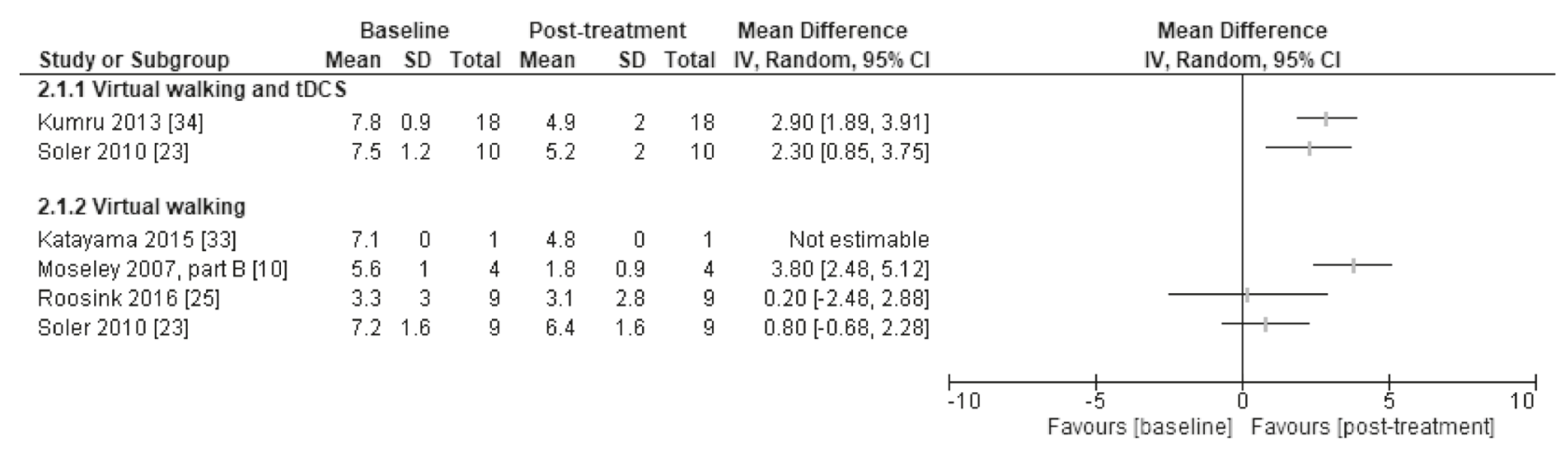

2.2 Comparing effect of $\mathrm{MI}$ on pain intensity at baseline and during intervention

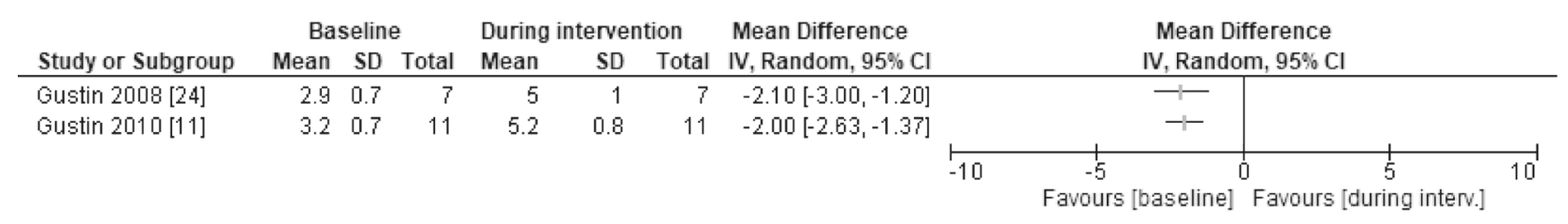

2.3 Comparing effect of MI to control intervention on pain intensity

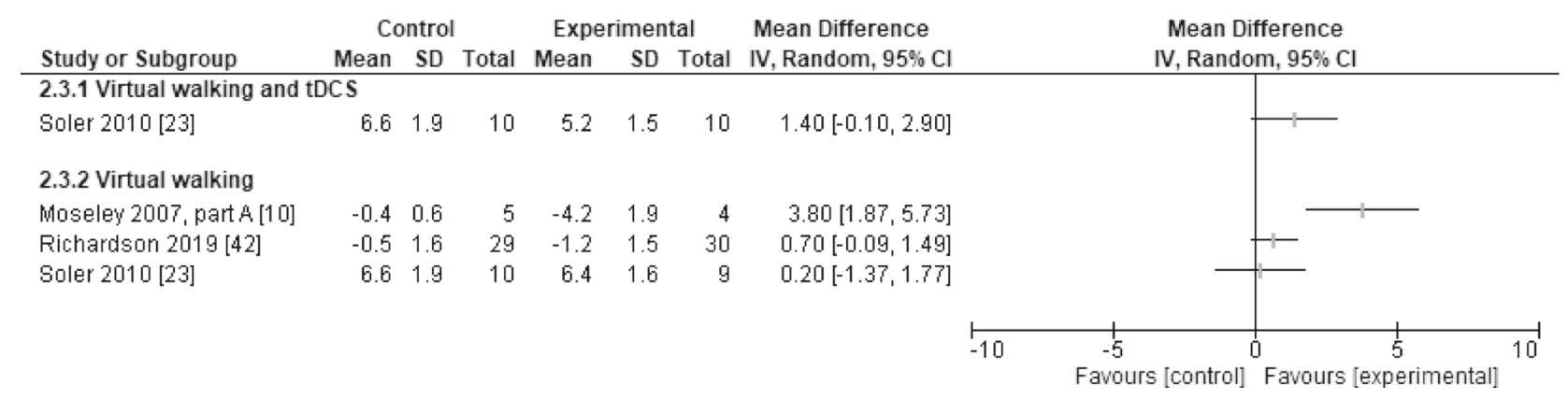

Fig. 2 Forests plots presenting the results of studies investigating the effect of motor imagery (MI) interventions on pain intensity in individuals with spinal cord injury. 2.1 Five studies were included, comparing the effect of an MI intervention on pain intensity at baseline and post treatment, which used either virtual walking (VW) combined with transcranial direct current stimulation (tDCS) (VW $+\mathrm{tDCS})$ or VW by itself. Three studies showed statistically significant results in favor of treatment by reducing pain and two showed nonsignificant

unpractised task. Sharp et al. [38] showed improvement in gait velocity both in a group using only overground training (OT) and in a group using OT in combination with MI. In their case study, Grangeon et al. [21] reported elbow extensor muscle scores increased by 1 point (maximal score 5) after MI (five sessions a week for 2 consecutive weeks). They also found an increased elbow amplitude associated with a decreased shoulder amplitude from pre-test to follow-up after MI and physical training [21]. Grangeon reduction of pain. 2.2 Two studies, comparing the effect of an MI intervention on pain intensity at baseline and during treatment, were included in this group, which had results showing an increase in pain intensity during MI. 2.3 Three studies were included comparing MI and a comparator intervention in terms of effect on pain intensity, with two in favor of the MI intervention. 95\% CI 95\% confidence interval, IV inverse variance, MI motor imagery, SD standard deviation, tDCS transcranial direct current stimulation, Total number of participants.

et al. [32] found that the movement time and trajectory smoothness of the upper limb improved following training and those measures remained stable after 3 months. Mateo et al. [35] showed a clinically significant improvement of wrist extension during tenodesis grasping after MI combined with usual rehabilitation, but no other effects on kinematics.

It was not possible to extract information about motor function outcomes after MI intervention separately for 
individuals with complete and incomplete SCI, or for tetraand paraplegic individuals, because these data were either pooled, not provided at all or there was a big discrepancy in the number of those with complete and incomplete SCI.

\section{Neurophysiological outcomes}

Several studies used neuroimaging, such as fMRI (with three-dimensional voxel analysis), to evaluate brain activity during MI intervention in individuals with SCI. It was shown that brain areas involved in movement control undergo reorganization after SCI [20]. Particularly, people with SCI, when compared with healthy individuals, showed the following changes: (1) extended activation volume in the left globus pallidus and posterior putamen - areas of the basal ganglia, which are involved in storage of learned motor sequences and in preparation for motor execution [45] and (2) spatial localization shifts of the primary sensory cortex activation area. Similar brain restructuring was reported in studies by Gustin et al. [11] and Mateo et al. [35], who observed a greater number of voxels activated in the supplementary motor area (involved in both initiation and inhibition of movements [46]), premotor cortex (involved in planning of movement [45]), and cerebellar cortex (involved in motor preparation, and particularly in inhibition of motor commands [45]) of individuals with SCI compared with healthy controls. It was also shown that MI training decreased the threshold for motor system activation after the application of transcranial magnetic stimulation [20]. Analysis of sensorimotor cortex activity using MEG showed that after MI intervention there was a decrease in brainwaves of beta frequencies $(13-35 \mathrm{~Hz}$, the range relevant for human brain motor processes) and in event-related desynchronization (ERD) parameters reflecting cortical excitation [35]. Some studies also reported dense neural connections between motor cortex and brain regions involved in pain processing $[11,34]$. In contrast to SCI patients without NeP, who had reduced ERD-EEG [35], SCI patients with $\mathrm{NeP}$ had an increase in ERD-EEG [40, 41]. This higher EEG activity in SCI patients with NeP was associated with better BCI performance than in those without NeP [36, 37, 40]. Evaluation of electro-dermal response duration (which positively correlates with motor cortex activity) during actual and imagined movement showed that SCI patients can perform MI as accurately as nondisabled people [32].

\section{Adverse events}

Eleven studies [10, 11, 20, 23-25, 34, 36, 37, 39, 40] mentioned adverse events but none listed serious adverse events. Two [37, 39] specified that there were no adverse events related to the intervention. An increase in pain was reported in five studies $[11,23-25,40]$ for 20 participants. For 15 participants $[11,24]$, the increase of pain intensity had already been reported as an outcome measure. The increase in pain was transient and pain returned to its preintervention intensity within $40 \mathrm{~min}$ after the intervention $[11,24]$. Unpleasant sensations (paresthesia, dysesthesia) were reported by eight participants [24, 40]. Other adverse events included headache $(n=4)[23,36]$, fatigue (at least ten participants) $[20,23,25,36]$, difficulty maintaining attention $(n=2)$ [25], mild transient postural hypotension $(n=1)$ [36], and distress during virtual walking $(n=1)[10]$.

\section{Discussion}

The objectives of this systematic review were to synthetize therapeutic benefits of MI on pain and motor function recovery in individuals with SCI, as well as to review neurophysiological outcome measures, and to describe the optimal type and dosage of MI intervention.

Although high heterogeneity in studies did not allow us to do meta-analysis and draw the firm conclusions, certain observations were made.

First, most of the findings confirmed the benefits of MI interventions on motor function when combined with physical practice $[20,32,35,38]$. The results from three RCTs [23, 38, 42] showed positive effects of MI as the sole treatment, on pain or motor function, but also when MI was used as an adjunct to other treatment (e.g., visual illusion, tDCS, or overground training).

Second, multiple repetitions and sessions of 30-60 min were used for motor function improvement $[20,21,27,32,35,38]$ and sessions of $8-20 \mathrm{~min}$ for pain treatment [10, 11, 23, 24, 33, 34, 39, 42].

Third, pain reduction was observed when comparing the effects within (but not across) the groups before and after MI intervention, whatever the protocol of MI treatment $[10,23,25,33,34,42]$. However, this was not the case in studies comparing pain before and during MI intervention $[11,24]$; then an increase in pain intensity was reported. There could be many reasons behind these results. For example, it is possible that MI, as well as the report of actual pain, is a complex cognitive process, which requires competencies and resources of the participant. Therefore, evaluation of actual pain should not be performed during MI [15, 47, 48]. Other causes might be related to the specifics of spinal cord lesion (i.e., NLI, severity, years since injury etc.), MI abilities of the individuals with chronic pain (in the presence of pain MI decreases over time [27]), and/ or type of pain assessment.

Indeed, imagery capacity of the participants with SCI was tested only in three out of 18 studies by using the KVIQ 
$[25,32,35]$. In those studies, general MI ability was very variable across the participants, ranging from poor to good, and with greater visual than kinesthetic imagery ability. These results point towards the importance of assessing imagery ability before an MI intervention as it could influence the results. In addition, we examined if the differences in pain outcome results between studies evaluating pain during and after MI intervention could be explained by NLI, the type and subtype of pain, or the pain assessment. The NLI was reported in all studies, except one [38] and varied. The pain type (nociceptive, neuropathic) and subtype (at or below level) were not always reported. Both NLI and pain subtype seem to have unclear impact on an MI intervention's effect on pain. Duration of pain (acute versus chronic) could also play role in MI interventions' effect. For example, a systematic review of different populations with musculoskeletal pain reported a significant MI effect only in those with chronic pain [49]. No information could be found in relation to the years with pain after SCI, but in one study participants with longer duration of SCI experienced a greater reduction in pain intensity from pre- to posttreatment [42]. In addition, there was a lack of consistent definitions of SCI pain categories in the studies included. Clear pain classification for individuals with SCI, as proposed in ISCIP (International Spinal Cord Injury Pain Classification), is considered an important step to correctly assess and treat the pain [2, 50]. Also, pain assessments across the studies were not the same. According to the Initiative on Methods, Measurement, and Pain Assessment in Clinical Trials recommendations about core outcome measures for chronic pain clinical trials [31], various dimensions of pain should be evaluated, such as pain intensity, pain quality, and the temporal components of pain.

In addition, other important outcomes of pain studies include physical and emotional functioning, patient satisfaction with treatment, symptoms, and adverse events [31]. For example, it was shown that anxiety and depression in individuals with SCI and pain [51] might affect MI performance [52-54]. However, in our systematic review we could not evaluate these psychosocial variables, as they were not explored in the studies retrieved.

When considering the effects of MI interventions on motor function, it could be important to test if different MI protocols should be applied for SCI individuals with complete and incomplete injury, tetra- or paraplegic patients. However, no conclusions could be drawn from the studies for the reasons of data heterogeneity or impossibility to extract results separately for individuals with complete and incomplete SCI. Similarly, only a few studies investigated the relationship between the completeness of SCI and pain outcome [23, 42]. They found no association between general pain changes [23] or changes in pain severity [42] and level of SCI (lumbar, thoracic, or cervical) or SCI severity (complete and incomplete). Others studies either did not consider this question or information could not be extracted.

Some studies included in this review examined neurophysiological measures to check brain activity when performing MI. Their data showed significant cortical reorganization after SCI [55, 56], when compared with nondisabled people. The changes were with respect to brain activation volume and patterns both during MI and movement execution. Particularly in people with SCI and NeP, dense neural connections were reported between motor cortex and brain regions involved in pain processing $[11,34]$. Interestingly, MI interventions reduced the number of recruited neurons, which could partially explain the motor function recovery and decrease in pain [32]. It was also observed that in contrast to SCI patients without NeP, those with $\mathrm{NeP}$ had higher EEG activity and better BCI performance [36, 37, 40]. However, it was not clear if this higher EEG resting state reflected abnormal activity in pain matrix brain circuitry, caused by cortical reorganization, or was a result of antidepressant and antiepileptic medication often taken by persons with SCI with NeP [40, 57].

\section{Conclusion}

Based on this systematic review, we cannot give detailed MI intervention guidelines or protocols for pain and motor function recovery in the SCI population. Only general observations can be offered, such as:

(1) It seems that when performing MI, pain outcome is not influenced by the level or severity of SCI.

(2) Shorter MI sessions were applied for pain reduction (average time of about $15 \mathrm{~min}$ based on reported range of 8-20 min) than for motor/functional improvement (average time of about $45 \mathrm{~min}$ based on reported range of 30-60 min). Therefore, to create guidelines more studies are needed with similar protocols with respect to population, intervention, and outcomes.

We think that the design of an MI intervention should also take into account the following factors, which were addressed by Milton et al. [58]: (1) complexity of the motor task and challenging environment are important to get better results, because conditions closer to a real world environment engage the motor system in an optimal way; (2) best performance requires attention to the assigned task as well as the ability to filter irrelevant information, which might be impaired in patients with nervous system diseases. In addition, before performing MI, it is crucial to test the imagery capacity of the participants [15] as neurological deficits may affect it [8]. 


\section{Future research}

There should be more studies comparing MI pain and motor function outcomes between individuals with complete and incomplete SCI. The effects of MI interventions on pain and its stability over time remains questionable. Therefore, clinical trials evaluating MI as standalone and/or adjunct therapy for NeP in SCI patients are warranted to develop appropriate guidelines for MI treatment.

\section{Data availability}

The datasets generated during the current study are available from the corresponding author upon request.

Funding The study was in part supported by a grant from the University of Applied Sciences and Arts Western Switzerland//HES-SO (scientific commission of health) to Emmanuelle Opsommer (73642/SRAD17-04).

Author contributions EOP and NKO were responsible for designing the review protocol, writing the protocol and report, conducting the search, and screening potentially eligible studies. EOP, OCH, and NKO were responsible for critically appraising studies, extracting and analyzing data, and interpreting results, updating reference lists, creating Summary of findings' tables, and writing the final report.

\section{Compliance with ethical standards}

Conflict of interest The authors declare that they have no conflict of interest.

Publisher's note Springer Nature remains neutral with regard to jurisdictional claims in published maps and institutional affiliations.

\section{References}

1. Finnerup NB. Pain in patients with spinal cord injury. Pain. 2013;154:S71-6.

2. Mahnig S, Landmann G, Stockinger L, Opsommer E. Pain assessment according to the International Spinal Cord Injury Pain classification in patients with spinal cord injury referred to a multidisciplinary pain center. Spinal Cord. 2016;54:809-15.

3. Siddall PJ, McClelland JM, Rutkowski SB, Cousins MJ. A longitudinal study of the prevalence and characteristics of pain in the first 5 years following spinal cord injury. Pain. 2003;103: 249-57.

4. Finnerup NB, Baastrup C. Spinal cord injury pain: mechanisms and management. Curr Pain Headache Rep. 2012;16:207-16.

5. Finnerup NB, Attal N, Haroutounian S, McNicol E, Baron R, Dworkin $\mathrm{RH}$, et al. Pharmacotherapy for neuropathic pain in adults: a systematic review and meta-analysis. Lancet Neurol. 2015;6:70251-0.

6. Tsubokawa T, Katayama Y, Yamamoto T, Hirayama T, Koyama S. Treatment of thalamic pain by chronic motor cortex stimulation. Pacing Clin Electrophysiol. 1991;14:131-4.

7. Le Dean Y, Brissebrat B, Castel-Lacanal E, De Boissezon X, Marque P. Management of neuropathic central pain by noninvasive brain stimulation and mirror therapy. Ann Phys Rehabil Med. 2016;59S:e145.
8. Di Rienzo F, Collet C, Hoyek N, Guillot A. Impact of neurologic deficits on motor imagery: a systematic review of clinical evaluations. Neuropsychol Rev. 2014;24:116-47.

9. Alkadhi H, Brugger P, Boendermaker SH, Crelier G, Curt A, Hepp-Reymond MC, et al. What disconnection tells about motor imagery: evidence from paraplegic patients. Cereb Cortex. 2005;15:131-40.

10. Moseley GL. Using visual illusion to reduce at-level neuropathic pain in paraplegia. Pain. 2007;130:294-8.

11. Gustin SM, Wrigley PJ, Henderson LA, Siddall PJ. Brain circuitry underlying pain in response to imagined movement in people with spinal cord injury. Pain. 2010;148:438-45.

12. Opsommer E, Korogod N. Mental practice for chronic pain in people with spinal cord injury: a systematic review protocol. JBI database Syst Rev Implement Rep. 2017;15:2004-12.

13. Barclay-Goddard RE, Stevenson TJ, Poluha W, Thalman L. Mental practice for treating upper extremity deficits in individuals with hemiparesis after stroke. Cochrane Database of Systematic Reviews. 2011;2011:CD005950.

14. Malouin F, Richards CL. Mental practice for relearning locomotor skills. Phys Ther. 2010;90:240-51.

15. Dickstein R, Deutsch J. Motor imagery in physical therapist practice. Phys Ther. 2007;87:942-53.

16. Schuster C, Hilfiker R, Amft O, Scheidhauer A, Andrews B, Butler J, et al. Best practice for motor imagery: a systematic literature review on motor imagery training elements in five different disciplines. BMC Med. 2011;9:75.

17. Hanakawa T, Immisch I, Toma K, Dimyan MA, Van Gelderen P, Hallett M. Functional properties of brain areas associated with motor execution and imagery. J Neurophysiol. 2003;89:989-1002.

18. Gentili R, Han CE, Schweighofer N, Papaxanthis C. Motor learning without doing: trial-by-trial improvement in motor performance during mental training. J Neurophysiol. 2010;104: 774-83.

19. Cocks M, Moulton CA, Luu S, Cil T. What surgeons can learn from athletes: mental practice in sports and surgery. $\mathrm{J}$ surgical Educ. 2014;71:262-9.

20. Cramer SC, Orr EL, Cohen MJ, Lacourse MG. Effects of motor imagery training after chronic, complete spinal cord injury. Exp brain Res. 2007;177:233-42.

21. Grangeon M, Guillot A, Sancho PO, Picot M, Revol P, Rode G, et al. Rehabilitation of the elbow extension with motor imagery in a patient with quadriplegia after tendon transfer. Arch Phys Med Rehabil. 2010;91:1143-6.

22. Braun S, Kleynen M, van Heel T, Kruithof N, Wade D, Beurskens A. The effects of mental practice in neurological rehabilitation; a systematic review and meta-analysis. Front Hum Neurosci. 2013;7:390.

23. Soler MD, Kumru H, Pelayo R, Vidal J, Tormos JM, Fregni F, et al. Effectiveness of transcranial direct current stimulation and visual illusion on neuropathic pain in spinal cord injury. Brain. 2010;133:2565-77.

24. Gustin SM, Wrigley PJ, Gandevia SC, Middleton JW, Henderson LA, Siddall PJ. Movement imagery increases pain in people with neuropathic pain following complete thoracic spinal cord injury. Pain. 2008;137:237-44.

25. Roosink M, Robitaille N, Jackson PL, Bouyer LJ, Mercier C. Interactive virtual feedback improves gait motor imagery after spinal cord injury: an exploratory study. Restor Neurol Neurosci. 2016;34:227-35.

26. Cohen SP, Mao J. Neuropathic pain: mechanisms and their clinical implications. BMJ. 2014;348:f7656.

27. Scandola M, Aglioti SM, Pozeg P, Avesani R, Moro V. Motor imagery in spinal cord injured people is modulated by somatotopic coding, perspective taking, and post-lesional chronic 
pain. J Neuropsychol. 2017;11:305-26. https://doi.org/10.1111/ jnp.12098.

28. Malouin F, Richards CL, Jackson PL, Lafleur MF, Durand A, Doyon J. The Kinesthetic and Visual Imagery Questionnaire (KVIQ) for assessing motor imagery in persons with physical disabilities: a reliability and construct validity study. J Neurol Phys Ther. 2007;31:20-9.

29. Aikat R, Dua V. Mental imagery in spinal cord injury: a systematic review. J Spine. 2016;5:1-8.

30. Institute JB. Joanna Briggs institute reviewers' manual. Adelaide: The Joanna Briggs Institute; 2014.

31. Dworkin RH, Turk DC, Farrar JT, Haythornthwaite JA, Jensen MP, Katz NP, et al. Core outcome measures for chronic pain clinical trials: IMMPACT recommendations. Pain. 2005;113: 9-19.

32. Grangeon M, Revol P, Guillot A, Rode G, Collet C. Could motor imagery be effective in upper limb rehabilitation of individuals with spinal cord injury? A case study. Spinal Cord. 2012;50: 766-71.

33. Katayama O, Iki H, Sawa S, Osumi M, Morioka S. The effect of virtual visual feedback on supernumerary phantom limb pain in a patient with high cervical cord injury: a single-case design study. Neurocase. 2015;21:786-92.

34. Kumru H, Soler D, Vidal J, Navarro X, Tormos JM, PascualLeone A, et al. The effects of transcranial direct current stimulation with visual illusion in neuropathic pain due to spinal cord injury: an evoked potentials and quantitative thermal testing study. Eur J Pain. 2013;17:55-66.

35. Mateo S, Di Rienzo F, Reilly KT, Revol P, Delpuech C, Daligault $\mathrm{S}$, et al. Improvement of grasping after motor imagery in C6-C7 tetraplegia: a kinematic and MEG pilot study. Restor Neurol Neurosci. 2015;33:543-55.

36. Onose G, Grozea C, Anghelescu A, Daia C, Sinescu CJ, Ciurea $\mathrm{AV}$, et al. On the feasibility of using motor imagery EEG-based brain-computer interface in chronic tetraplegics for assistive robotic arm control: a clinical test and long-term post-trial followup. Spinal Cord. 2012;50:599-608.

37. Salisbury DB, Parsons TD, Monden KR, Trost Z, Driver SJ. Brain-computer interface for individuals after spinal cord injury. Rehabil Psychol. 2016;61:435-41.

38. Sharp KG, Gramer R, Butler L, Cramer SC, Hade E, Page SJ. Effect of overground training augmented by mental practice on gait velocity in chronic, incomplete spinal cord injury. Arch Phys Med Rehabil. 2014;95:615-21.

39. Sumitani M, Miyauchi S, McCabe CS, Shibata M, Maeda L, Saitoh Y, et al. Mirror visual feedback alleviates deafferentation pain, depending on qualitative aspects of the pain: a preliminary report. Rheumatol. 2008;47:1038-43.

40. Vuckovic A, Hasan M, Osuagwu B, Fraser M, Allan D, Conway $\mathrm{B}$, et al. The influence of central neuropathic pain in paraplegic patients on performance of a motor imagery based Brain Computer Interface. Clin Neurophysiol. 2015;126:2170-80.

41. Xu R, Jiang N, Vuckovic A, Hasan M, Mrachacz-Kersting N, Allan D et al. Movement-related cortical potentials in paraplegic patients: abnormal patterns and considerations for BCIrehabilitation. Front Neuroeng. 2014;7:35. https://doi.org/10. 3389/fneng.2014.00035.

42. Richardson EJ, McKinley EC, Rahman A, Klebine P, Redden DT, Richards JS. Effects of virtual walking on spinal cord injury- related neuropathic pain: a randomized, controlled trial. Rehabil Psychol. 2019;64:13-24.

43. Siddall P, Yezierski BKJR, Loeser JD. Taxonomy and epidemiology of spinal cord injury pain. In: Yezierski B, editor. Progress in pain research and management. vol. 23. Seattle: IAP Press; 2002. p. 9-23.

44. Bryce TN, Ragnarsson KT. Pain after spinal cord injury. Phys Med Rehabil Clin North Am. 2000;11:157-68.

45. Guillot A, Di Rienzo F, Collet C. The neurofunctional architecture of motor imagery. In: Advanced brain neuroimaging topics in health and disease-methods and applications. London: IntechOpen; 2014. p. 433-56. https://www.intechopen.com/books/a dvanced-brain-neuroimaging-topics-in-health-and-diseasemethods-and-applications/the-neurofunctional-architecture-ofmotor-imagery.

46. Kasess $\mathrm{CH}$, Windischberger $\mathrm{C}$, Cunnington $\mathrm{R}$, Lanzenberger R, Pezawas L, Moser E. The suppressive influence of SMA on M1 in motor imagery revealed by fMRI and dynamic causal modeling. NeuroImage. 2008;40:828-37.

47. Ferchichi S, Opsommer E. La pratique mentale pour la rééducation suite à un accident vasculaire cérébral. Un complément aux interventions conventionnelles pour la récupération de la fonction. Kinésithér Rev. 2015;15:38-44.

48. Jensen MP, Karoly P. Self-report scales and procedures for assessing pain in adults. In: Turk DC, Melzack RE, editors. Handbook of pain assessment. New York, NY, US: The Guilford Press; 2011. p. 11-44.

49. Yap BWD, Lim ECW. The effects of motor imagery on pain and range of motion in musculoskeletal disorders: a systematic review using meta-analysis. Clin J Pain. 2019;35:87-99.

50. Bryce TN, Biering-Sorensen F, Finnerup NB, Cardenas DD, Defrin R, Lundeberg $\mathrm{T}$, et al. International spinal cord injury pain classification: part I. Backgr Description Spinal Cord. 2012;50: 413-7.

51. Landmann G, Berger MF, Stockinger L, Opsommer E. Usefulness of laser-evoked potentials and quantitative sensory testing in the diagnosis of neuropathic spinal cord injury pain: a multiple case study. Spinal Cord. 2017;55:575-82.

52. Tabrizi YM, Mazhari S, Nazari MA, Zangiabadi N, Sheibani V. Abnormalities of motor imagery and relationship with depressive symptoms in mildly disabling relapsing-remitting multiple sclerosis. J Neurol Phys Ther. 2014;38:111-8.

53. Bennabi D, Monnin J, Haffen E, Carvalho N, Vandel P, Pozzo T, et al. Motor imagery in unipolar major depression. Front Behav Neurosci. 2014;8:413.

54. Thomschewski A, Strohlein A, Langthaler PB, Schmid E, Potthoff $\mathrm{J}$, Holler $\mathrm{P}$, et al. Imagine there is no plegia. mental motor imagery difficulties in patients with traumatic spinal cord injury. Front Neurosci. 2017;11:689.

55. Harris AJ. Cortical origin of pathological pain. Lancet. 1999;354: 1464-6.

56. Haanpää M, Attal N, Backonja M, Baron R, Bennett M, Bouhassira D, et al. NeuPSIG guidelines on neuropathic pain assessment. PAIN. 2011;152:14-27.

57. Olbrich S, Arns M. EEG biomarkers in major depressive disorder: discriminative power and prediction of treatment response. Int Rev Psychiatry. 2013;25:604-18.

58. Milton J, Small SL, Solodkin A. Imaging motor imagery: methodological issues related to expertise. Methods. 2008;45:336-41. 